\title{
Study on the System Design of Campus Resource Sharing Platform
}

\author{
Zeng $\mathrm{Xi}^{1,1 \mathrm{st}}, \quad \mathrm{Zhou} \mathrm{Yu^{1,a,2nd }}$, Chen Xuqi ${ }^{1,3 r d}$ \\ ${ }^{1}$ Wuhan Institute of technology, School of Art and Design, Wuhan University of Technology, Wuhan City, China
}

\begin{abstract}
The development of the times and the development of the Internet have changed people's consumption concepts. The author has conducted user research on user groups such as college students. After data analysis, the demand for resource sharing and trading of second-hand goods of college students was transformed into the function point of the trading platform. The APP was designed and developed to solve the needs of college students to share and exchange resources on campus. The APP's main functions are secondhand commodity trading and virtual resource trading.
\end{abstract}

\section{Introduction}

Changes in the times have led to changes in people's ideology and behavioral needs, and the concept of sustainable consumption has gradually been accepted. With the development of society and the improvement of people's living standards, the upgrading and upgrading of college students' items has accelerated, especially when college graduates are facing the difficulty of handling a large number of items near graduation. In this social context, the huge potential of the secondary market on university campuses is obvious. In many western developed countries, the forms and transactions of used objects are very flexible. Today 's people 's understanding of second-hand goods has changed a lot. They believe that the "secondary circulation" of goods makes social resources effective. Utilization is conducive to sustainable development, and reproduction and re-consumption will become a trend and trend [1]. With various forms of student activities such as exchange of experience at various levels of examinations, postgraduate and postgraduate training, study abroad counseling, part-time internships, and business promotion activities and other information continuously enrich the campus life of college students, the demand situation of college students has evolved into the demand for physical objects. There is also a need for the aforementioned virtual campus resources. The author conducts an empirical analysis through questionnaires and interviews to clarify the feasibility of a mobile app for mutual placement of college campus resources, and points out the direction for the design of the app.

\section{Status of Campus Resource Sharing Needs}

In order to investigate the current situation of college students' demand for campus material resources and virtual resources in campus life (including the purpose of access to campus resources, the channels and the new characteristics of the types of campus resources obtained), the author uses the Wuhan University of Engineering undergraduates of different grades as the study subjects, and conducts the relevant research by means of anonymous questionnaires and interview research. The questionnaire was set up with closed questions (optional questions), supplemented by open questions (no optional questions), and a total of 310 questionnaires were distributed on the campus of Wuhan University of Engineering, 310 were collected, and 310 valid questionnaires were collected, with a $100 \%$ efficiency. The number of respondents to the closed questions was 310 and the number of respondents to the open questions was 289 [2].

A specific questionnaire was prepared for the student population of Wuhan University of Engineering, with reference to the questionnaires on the second-hand transactions of university students on various Internet platforms, and a qualitative classification of all evaluation items was carried out. The initially developed topics was being discussed with the instructors, six undergraduate students and two graduate students to further define and refine their topics. The questionnaire on the current status of resource sharing on college campuses was finally developed after repeated refinement and revision.

From the attitude of the respondents to dispose of idle items, most of the respondents in the valid questionnaire results of the recycling indicated that there is no convenient and quick way to dispose of idle items on 
campus, so they will take the disposal method to solve the problem, which shows that there is a great market development prospect of creating campus resource sharing platform.

From the respondents have used the second-hand trading platform in the distribution of statistics (multiple choice) can be seen, having used the "XianYu " APP accounted for the vast majority, accounting for $76.98 \%$, having used the "Zhuan Zhuan " of up to $4.76 \%$, having used the "Post Bar " APP accounted for $11.9 \%$, having used the campus QQ group accounted for $40.48 \%$, having used Taobao to buy second-hand goods accounted for $35.71 \%$, other ways accounted for $6.35 \%$. Through the second-hand trading platforms that the respondents have used, it can be found that the main way for college students to buy second-hand items at this stage is "Xian Yu" APP, and there is no platform for college students to use, which also shows the market potential of creating campus resource sharing platforms.

In the survey, the respondents indicated that the demand for learning resources (multiple choices) in campus life is mainly interested in hobbies (painting, musical instruments, sports, etc.) accounted for $59.06 \%$, future planning (examinations, employment, experience abroad, etc.) accounted for $48.03 \%$, examinations and grades (CET4\&6, TOEFL IELTS, computing L2, accounting certificate, etc.) accounted for $48.82 \%$, and usual class assignments (advanced mathematics, physics, macro classes, etc.) accounted for $30.7 \%$. At the same time, the vast majority of respondents expressed a willingness to trade skills in exchange for money. It can be seen that while college students meet the demand for physical transactions, they also have a demand for virtual resources, the main demand for virtual resources are for learning experiences and learning skills. It demonstrates the market potential for developing a platform that brings together physical and virtual objects to share resources on college campuses.

\section{System Architecture Design}

\subsection{Overall System Architecture Design}

Since the development and design direction is the university campus resource sharing platform, the user group is mainly the university students who have the need to buy second-hand goods or study skills, interchange skills and share learning resources.

In order to solve the problems that college students may encounter in the process of resource interposition, the author has designed a convenient and secure service platform for the interposition of resources on college campuses - "GUAPO" APP, the functional structure of which is shown in Figure 1.

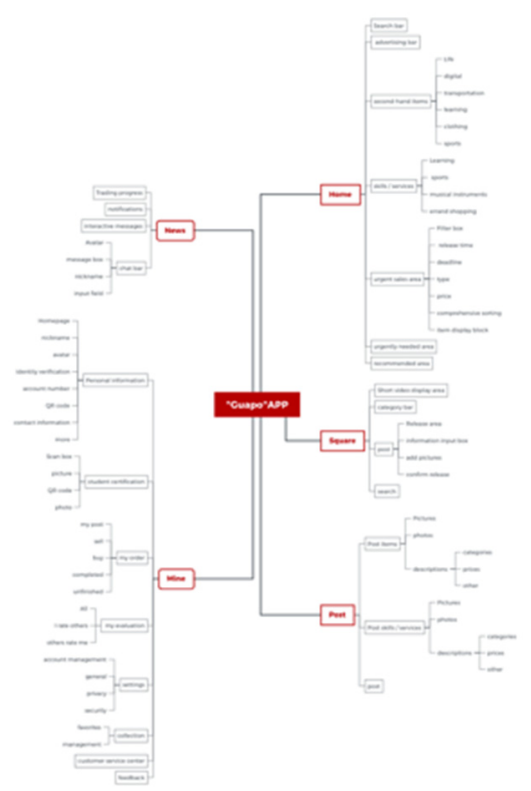

Fig1. Design functional structure diagram

\subsection{Function Design}

For the design of the interchangeable platform for university students' campus resources in the program development process, from the user research process to obtain the design of APP function points, there are the following modules to be designed.

\subsubsection{Posting and item trading module design}

From the user research, we can conclude that college students have an excellent demand for virtual resources such as learning skills and learning experience even though they have a high desire for physical objects. Respondents indicated in the interview that the demand for learning resources (multiple choices) in campus life is mainly interested in hobbies (painting, musical instruments, sports, etc.) accounting for $59.06 \%$, and personal development plan (postgraduate, employment, study abroad, etc.) accounted for $48.03 \%$, grade examinations (CET4\&6, TOEFL, IELTS, computing level 2 , accounting certificate, etc.) accounted for $48.82 \%$, academic tasks (advanced mathematics, physics, etc.) accounted for $30.7 \%$, competition guidance ( innovation and entrepreneurship competition, mathematical modeling competition, interdisciplinary competition, etc.) accounted for $29.13 \%$. Meanwhile, the vast majority of respondents alleged their willingness to replace money transactions utilizing skills exchange. It can be concluded that while college students satisfy the demand of physical resource transactions, they also need virtual resources, especially learning experience and learning skills. The above points can prove that there is great potential for the development of a market that integrates physical and virtual college students' campus resource sharing platforms. Therefore, the GUAPO APP is divided into two different function points: physical goods area and virtual goods area. When users use the GUAPO APP, they first select posting on the home page, then select the type of posting as physical goods or virtual resources such as 
skills and services, then select the transaction time, edit the content and finally publish the message. The operational flow and low-fidelity interaction design are shown in Figure 2.
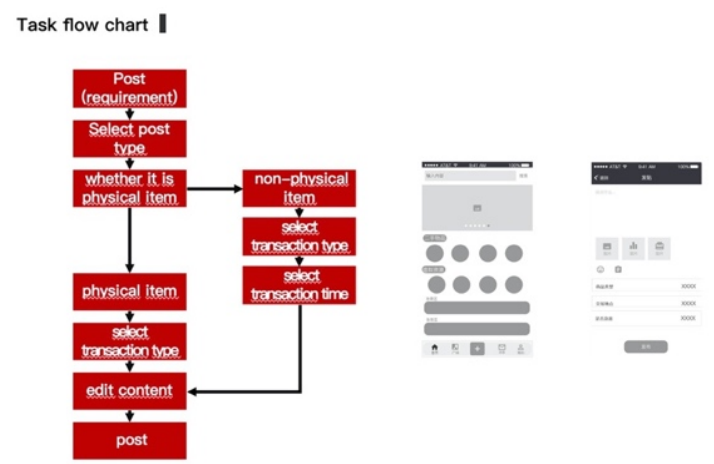

Fig2. The operation process and Lo-Fi prototype of "GUAPO" by author.

The seller users, when using the platform, want to sell items and skills first click on the home page, select the release time or skills, then fill in the commodity information, upload pictures or video information, confirm the transaction method. Also, the rush sale function was added to meet sellers' demand for quick-toshoot items, as shown by the sellers' operating procedures and low fidelity (Figure 3).

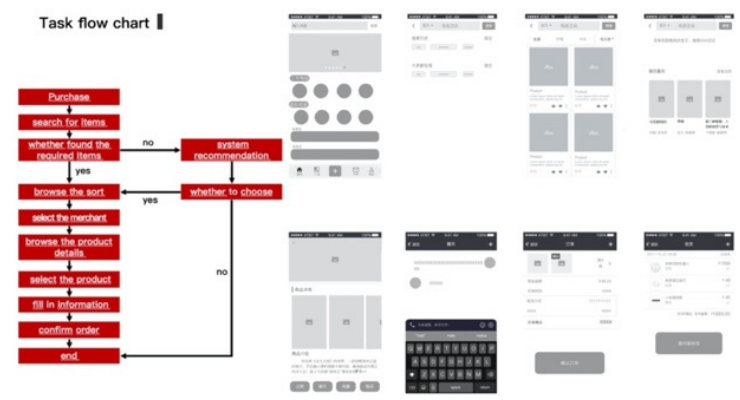

Fig3. The operation process and Lo-Fi prototype of "GUAPO" by author.

When the seller wants to sell items or skills on the platform, firstly click the publishing button on the home page to update the goods, then select to publish time or items, thirdly fill in the item information and upload pictures or videos about issues, finally confirm the transaction method. At the same time, the urgent sale function has been designed to satisfy the seller's demand. The seller's operation process and Lo-Fi prototype are shown in the figure. (Figure 4)
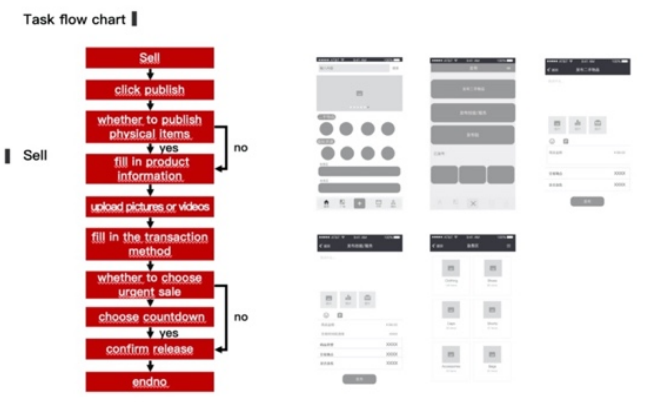

Fig4. The operation process and Lo-Fi prototype of "GUAPO" by author.

\subsubsection{Student certification module design}

In the course of user research, we learned that the majority of college students encounter problems with the quality of items that do not match the seller's descriptions when conducting second-hand transactions. From the problems respondents encountered in using the second-hand trading platform (multiple choices), it can be found that respondents generally encountered the following three kinds of problems: $14.96 \%$ of those who encountered problems with damage in logistics, poor quality of goods (service), inconsistency between buyers' show and sellers' show as high as $48.82 \%$, return of goods, warranty inconvenience accounted for $44.88 \%$, other problems accounted for $34.65 \%$. Analysis from this question reveals that most of the existing platforms on the market have problems such as the quality and safety. Therefore, the "GUAPO" APP adopts a closed school-to-school connection so that only students in school can trade with students in this school, which can ensure that students can obtain the required items and quality inspection of the items in time when trading. At the same time, most users who buy second-hand items will experience the issue of distance and not being able to inspect the goods. Therefore, "GUAPO" APP adopts campus card authentication and registration to ensure that the user's real identity is a college student, and the transaction with the same school can ensure the resolution of transaction disputes and the convenience of seeing the goods transaction, while eliminating the logistics costs. This setting of transactions between students at the same university is also intended to ensure the existence of post-sales disputes and the personal safety of the transaction process, as shown in the student authentication module process (Figure 5).
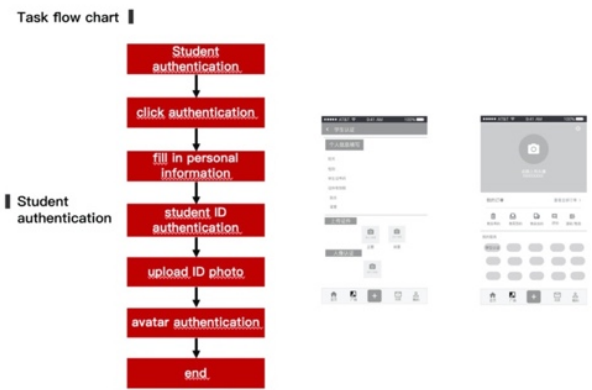

Fig5. The operation process and Lo-Fi prototype of "GUAPO" by author.

\subsection{System high fidelity design implementation}

After the development of the "GUAPO" APP system can run normally on the mobile phone, the user interface needs further improvement, part of the system interface functions is shown in the following figure. Tested by functional testing and usability quizzes. After analyzing the above-mentioned test results, the campus resource sharing system developed by this platform meets the requirements and needs of the clients. All the basic features are complete, easy to operate, the product runs well and is a mass system worth promoting. According to the clustering analysis of the platform's user data, users with similar behaviors are clustered into a cluster, their 
behavioral habits are analyzed, and users are profiled, so that specific resource information is pushed to the same type of users, making resource utilization more efficient [3].

\subsection{Visual design principles and high-fidelity interface}

The APP has the modules of home page, publishing, square, user authentication, etc. The interface color is mainly green and white, with a fresh and simple feeling, which is in line with the university students. The font is in Microsoft Ebony, with a font size of $28 \mathrm{px}$ as the standard, ranging from $36 \mathrm{px}$ to $22 \mathrm{px}$ depending on the importance of the text (see Figure 6).

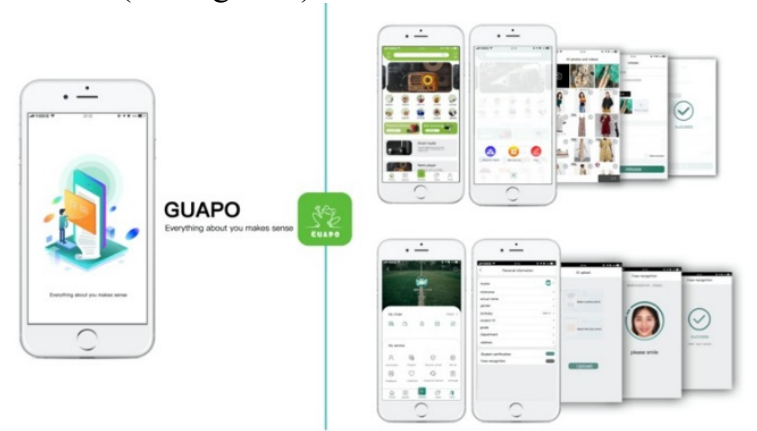

Fig6. The High-fidelity interface design of "GUAPO."

\subsection{Usability testing}

\subsubsection{User Portrait}

In the usability test, the author divided the users into three groups according to their different needs for the product: first, users with high frequency demand for campus resource interchange; second, users with low frequency demand for campus resource interchange; third, users with demand for campus resource interchange in special time node. The author selected college students of different ages, genders, shopping characteristics and needs to build three user profiles.

\subsubsection{User transaction process}

The author extracted one from the three user portraits and simulated the process when using the campus resource sharing platform, depicting the four stages of browsing, picking, buying and receiving from users using the platform (as shown in Figure 7).

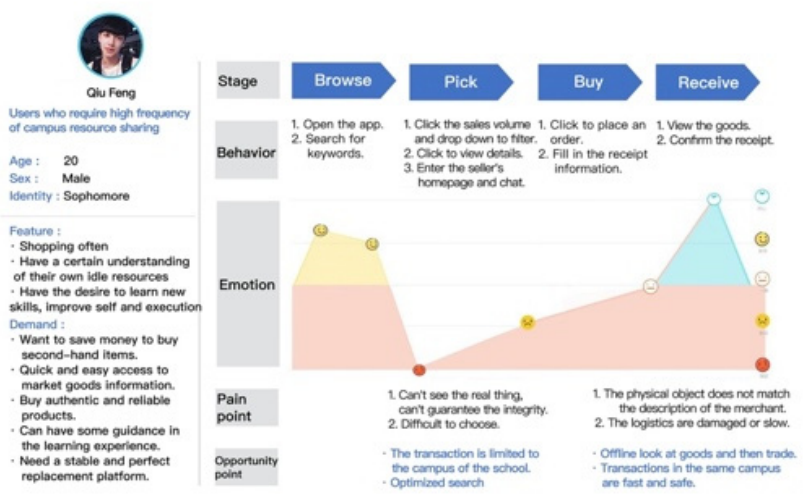

Fig7. User transaction process

\subsubsection{Storyboard}

The author has drawn a storyboard to depict the scenario where students are using "GUAPO" to obtain learning resources: A college student wanted to learn guitar, but the course was not only far away but expensive, so he opened the "GUAPO" app, found a student on the platform who is proficient in playing the guitar and posted a video. After online contacting, they met offline and had a deal to learn guitar two hours a week, finally the student acquired the resources he wanted by "GUAPO." (Figure 8)
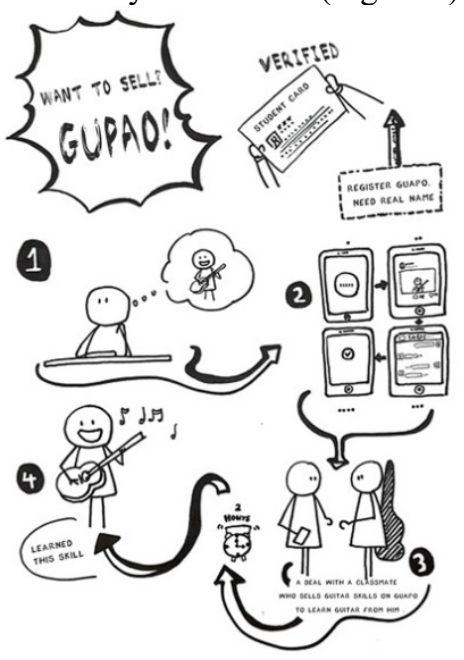

Fig8. Storyboard Explanation

\subsection{Usability Testing Summary of Chapter 3}

Through user demand analysis, it is possible to identify the huge potential of the market for resource sharing platforms for college campuses and the preferences and attitudes of users towards such platforms. The following is a feasibility analysis.

- Market viability: the resources of university campuses are promising, and the consumer base is very large and constantly changing.

- Operational feasibility: to be able to penetrate into the life of college students, the flow of $4 \mathrm{G}$ network makes its spread in the campus fast, convenient operation saves college students a lot of time. 
- Economic viability: more access to market demand for campus resources and lower cost.

- Regulatory feasibility: buyers and sellers trade independently and in a variety of ways.

\section{Conclusion}

Designers are always exploring how they can create designs that feel good to the user. The innovative thinking of designers is no longer limited to the design of a product, but lies in making humanized design, to integrate various resources and the use of scientific and technological means to meet the growing needs of users, to build a more complete product service experience. The development of mobile terminals and Internet technology has prompted the use of mobile applications to become a trend, but the domestic second-hand trading market has not been fully tapped, and there is no complete campus resource sharing platform for university students. In this article, from the perspective of system design, combined with real research results, we identify user pain points and opportunity points, build a complete system to develop APP and provide the functional modules that users need.

\section{Acknowledgment}

First of all, I would like to thank my mentor for the academic guidance and shortcomings that I have provided in my scientific research life, so that I can continue to progress. Secondly, I would like to thank the students who participated in this design project for their innovative ideas and design assistance. Finally, I would like to thank Mr. Zhu for modifying the text of this paper so that I can complete this article.

\section{References}

1. Huang Jieying, Ge Wei, Li Xiujie, Gao Bin, Ding Yingbin, Zhao Shuang. User demand analysis of second-hand trading mobile APP [J]. Communications World, 2016(06):244-245.

2. Li Dandan. Investigation and Research on Teachers, Professional Qualities of Primary School General Teachers[D]. Sichuan Normal University, 2018.

3. Wang Mengmeng, Zeng Fangui. Research on Product Design of Resource Sharing App under Service Design Thinking - - Taking Help $\mathrm{Me}$ as an Example[J].Arts and Design (Theory),2017,2(06):9799. 